\title{
Cysteine-rich 61 (CYR61) inhibits cisplatin-induced apoptosis in ovarian carcinoma cells
}

\author{
Seung Bae Rho · Jong Soo Woo • \\ Taehoon Chun · Sang-Yoon Park
}

Published online: 5 November 2008

(C) Springer Science+Business Media B.V. 2008

\section{Erratum to: Biotechnol Lett \\ DOI 10.1007/s10529-008-9845-8}

The list of authors and the Acknowledgements section in the original publication were incorrect. The list of authors in this erratum is correct. The correct Acknowledgements section is shown below.

Acknowledgements This work was supported by a grant from the National Cancer Center, Korea (NCC-0810410-1). We thank Dr. Hyun-Jung Byun (Korea University, Republic of Korea) for critical reading of the manuscript.

The online version of the original article can be found under doi:10.1007/s10529-008-9845-8.

S. B. Rho $(\bowtie) \cdot$ J. S. Woo

Research Institute, National Cancer Center, 809,

Madu 1-dong, Ilsan-gu, Goyang-si, Gyeonggi-do 411-769,

Republic of Korea

e-mail: sbrho@ncc.re.kr

T. Chun

Division of Biotechnology, College of Life Sciences and Biotechnology, Korea University, Seoul 136-701, Republic of Korea

S.-Y. Park

Center for Uterine Cancer, Research Institute and

Hospital, National Cancer Center, 809, Madu 1-dong,

Ilsan-gu, Goyang-si, Gyeonggi-do 411-769,

Republic of Korea 